\title{
STUDIES ON BAMBARA GROUNDNUT (Vigna subterranea (L.)Verdc.) INDUCED WITH SODIUM AZIDE USING SODIUM DODECYL SULPHATE POLYACRLYAMIDE GEL ELECTROPHORESIS (SDS-PAGE)
}

\author{
Juliet Naworu ${ }^{1}$ \\ ${ }^{1}$ University of Ibadan Faculty of Science
}

September 28, 2020

\begin{abstract}
This study investigated the mutagenic tolerance of eight Bambara groundnut genotypes to Sodium azide (NaN3) using SDSPAGE. The seeds of six genotypes; TVSu-86, TVSu-91, TVSu-186, TVSu-235, TVSu-242, TVSu-350 were collected from IITA and two landraces from Abia and Enugu local markets. The seeds were treated with five concentrations: $0.00 \%$ (control), $0.01 \%$, $0.03 \%, 0.05 \%$ and $0.07 \%$ of NaN3 after pre-soaking for $6 \mathrm{hrs}$ in distilled water and sown in pots arranged in a Complete Randomized Design with three replicates. There was reduction in germination percentage and growth characters as concentrations of NaN3 increases. Early flowering was recorded at 37 days mutated with $0.07 \%$ of NaN3 compared to control which flowered late at 42 days. NaN3(0.07\%) caused lethal effect on Abia and Enugu landraces. There was no significant $(\mathrm{P}>0.05)$ difference in yield traits among mutants and control. Mutant seeds significantly $(\mathrm{P}<0.05)$ increased protein content $(19.12 \%)$ at $0.05 \%$ of NaN3 compared to control(18.5\%). The number of seeds(0.99), seed yield(0.89) and pod yield(0.96) strongly correlated with seeds per pod (0.85). The SDS-PAGE revealed the presence of polypeptide bands in mutants compared to control. TVSu-235 and TVSu-350 genotypes had higher tolerance and yield traits to $0.01 \%$ concentration of NaN3, thus could be further improved in subsequent breeding.
\end{abstract}

\section{INTRODUCTION}

Bambara groundnut (Vigna subterranea (L.)Verdc,), member of the family Fabaceae is an indigenous, underutilized African legume crop mainly grown by subsistence farmers for food (Bamishaiye et al ., 2011). Bambara groundnut is the third most important leguminous crop after Groundnut and Cowpea in terms of production and consumption with which it shares its origin of genetic diversity (Aremu et al., 2006). Genetic variation is fundamental to successful breeding programs in vegetatively and sexually propagated plants (Olawuyiet al ., 2015; Agbolade et al ., 2016). This variation which occurs naturally or artificially by inducing with physical, biological or chemical mutagens has attracted the interest of plant breeders for many decades. Mutation breeding is an aspect of conventional plant breeding which involves physical and chemical mutation in order to assess genetic variation resulting to improved varieties with better characteristics (Wongpiyasatid et al ., 2000; Mensah and Obadoni, 2007; Arulbalachandran et al ., 2009; Olawuyi et al ., 2016).

Among numerous techniques available for assessing the genetic variability and relatedness among crop germplasm, seed storage protein analysis represents a valid alternative and improved approach to varietal identification (Mennella et al ., 1999). Iqbal et al . (2005) reported that grain storage protein profiling based on SDS-PAGE can be employed for various purposes such as; characterization of germplasm, varietal 
identification, biosystematics analysis, determination of phylogenetic relationship between different species and generation of pertinent information to complement evaluation (Ghafooret al ., 2002).

Sodium azide $\left(\mathrm{NaN}_{3}\right)$ is one of the constituents of bactericide, pesticide and industrial nitrogen gas generator that may be lethal or beneficial in several plants such as barley, maize and some other crop species (Ilbas et al ., 2005; Mostafa, 2011).

Therefore, this study investigated the effects of sodium azide on morphological and protein contents of Bambara groundnut using SDS-PAGE.

\section{MATERIALS AND METHODS}

Collection of seed samples, experimental location and experimental design

Six (6) Seeds of Bambara Genotypes: TVSu-86, TVSu-91, TVSu-186, TVSu-235, TVSu-242, TVSu-350 were collected from the germplasm unit of the International Institute of Tropical Agriculture (IITA), Ibadan, Nigeria and two (2) landraces were collected from Abia State and Enugu State, Nigeria.

The in vitro and screen house experiments were carried out at the Genetics and Molecular Laboratory and Nursery farm of the Department of Botany, University of Ibadan, Nigeria respectively. The design was laid out in Complete Randomized Design (CRD) comprising of 6 genotypes, 2 landraces, 5 concentrations and 3 replicates.

Preparation of sodium azide $\left(\mathrm{NaN}_{3}\right)$, pre-treatment of seeds and in vitro screening

The seeds of Bambara were treated with concentrations of sodium azide $(0.01 \%, 0.03 \%, 0.05 \%$ and $0.07 \%)$ after pre-soaked in $100 \mathrm{ml}$ of distilled for $\operatorname{six}(6)$ hours at room temperature $\left(25^{\circ} \mathrm{C}\right)$, while untreated seeds $(0.00 \%)$ served as control according to the method described by Asad et al. (2014). Five seeds of both treated and pre-soaked seeds were blotted with Whatman filter paper to remove excess moisture for 20minutes and planted on Petri dishes lined with moist filter paper. $2 \mathrm{ml}$ of distilled water was added daily to the petri dish according to the method described by Asgharipour and Rafiei (2011).

Research design and Screen house experiment

$10 \mathrm{~kg}$ of sterilized top soil was measured using a weighing scale and put into 120 perforated polythene bags. Three treated and untreated seeds of each Bambara genotype were sown 3 inches deep inside polythene bag filled with sterilized soil and spaced at a distance of $30 \mathrm{~cm}$ in a completely randomized design (CRD). Watering and other agronomic practices were also carried out during growth and development of the plant until maturity.

Determination of Quantitative characters and Protein contents

Observations on morphological traits of $\mathrm{NaN}_{3}$ induced mutants and controls were carried out and measured using measuring tape and visual counting for a period of 14 weeks after planting following the IPGRI, IITA and BAMNET (2000) Descriptors for Bambara groundnut. The crude protein content was determined using micro Kjeldahl method as described in AOAC (1996) and Sodium Dodecyl Sulphate - Polyacrylamide Gel Electrophoresis (SDS-PAGE)was analysed using $12 \%$ vertical slab polyacrylamide gel for the separation of polypeptide bands according to the procedure described by Weber and Osborn (1975).

\section{Statistical analysis}

Data were analyzed by analysis of variance (ANOVA) using SAS generalized linear model (GLM) software and means with significant differences were separated by Duncan multiple range test (DMRT) (P[?]0.05).The final germination percentage (FGP) was obtained using Cokkizgin and Cokkizgin (2010) method as stated below;

$$
\mathrm{FGP}=\frac{\text { number of germinated seeds after } 8 \text { days }}{\text { total number of seeds planted }} \times 100
$$




\section{RESULTS AND DISCUSSION}

There was reduction in the germination percentage and growth characters as the concentration of sodium azide increases. This could be as a result of the lethal effect caused by the mutagen. Similar reductions were also observed in maize in the reports made by Olawuyi and Okoli (2017).

Table 1. Mean square interaction of genotype, concentrations of Sodium azide and growth stage on germination and growth characters of Bambara groundnut

\begin{tabular}{llllll}
\hline Source of variation & Df & Germination percentage & Plant height & Leaf length & Number of leaves \\
\hline Concentration & 4 & $9.69^{\mathrm{ns}}$ & $2121.02^{* * *}$ & $134.37^{* * *}$ & $42476.98^{* * *}$ \\
Genotype & 7 & $385.48^{* * *}$ & $3321.67^{* * *}$ & $274.36^{* * *}$ & $143861.73^{* * *}$ \\
Replicate & 2 & $2.32^{\mathrm{ns}}$ & $60.89^{\mathrm{ns}}$ & $11.92^{\mathrm{ns}}$ & $5453.61^{\mathrm{ns}}$ \\
Week & 13 & $17146.10^{* * *}$ & $34.80^{* * *}$ & $2.07^{\mathrm{ns}}$ & $91419.258^{* * *}$ \\
Genotype $\times$ concentration & 24 & $87.58^{\mathrm{ns}}$ & $892.50^{* * *}$ & $52.29^{* * *}$ & $26745.69^{* * *}$ \\
Concentration $\times$ replicate & 8 & $74.82^{\mathrm{ns}}$ & $120.31^{* * *}$ & $5.18^{* * *}$ & $25610.86^{* * *}$ \\
Concentration $\times$ week & 52 & $99.59^{* * *}$ & $30.14^{* * *}$ & $2.18^{* * *}$ & $1828.05^{* * *}$ \\
Genotype $\times$ replicate & 14 & $73.01^{\mathrm{ns}}$ & $66.85^{* * *}$ & $3.29^{* * *}$ & $3478.95^{* * *}$ \\
Genotype $\times$ week & 91 & $385.60^{* * *}$ & $47.53^{* * *}$ & $3.61^{* * *}$ & $3786.25^{* * *}$ \\
Week $\times$ replicate & 26 & $10.52^{\mathrm{ns}}$ & $11.59^{\mathrm{ns}}$ & $0.69^{\mathrm{ns}}$ & $539.00^{\mathrm{ns}}$ \\
Genotype $\times$ concentration $\times$ replicate & 47 & $45.91^{\mathrm{ns}}$ & $117.29^{* * *}$ & $10.33^{* * *}$ & $13250.09^{* * *}$ \\
Genotype $\times$ concentration $\times$ week & 312 & $87.58^{* * *}$ & $32.89^{* * *}$ & $3.05^{* * *}$ & $2166.30^{* *}$ \\
Concentration $\times$ week $\times$ replicate & 104 & $74.82^{* * *}$ & $15.29^{\mathrm{ns}}$ & $1.52^{*}$ & $1629.64^{* * *}$ \\
Genotype $\times$ week $\times$ replicate & 182 & $73.01^{* * *}$ & $18.88^{*}$ & $1.39^{\mathrm{ns}}$ & $1094.67^{\mathrm{ns}}$ \\
Error & 611 & 611 & 562 & 562 & 560 \\
Corrected total & 1497 & 1497 & 1446 & 1446 & 1444 \\
\hline
\end{tabular}

Note: ${ }^{*}=$ significant at $p<0.05,{ }^{* *}=$ highly significant at $p<0.01,{ }^{* * *}=$ highly significant at $p<0.001$

Early flowering was recorded in Bambara groundnut mutated with $0.07 \%$ of sodium azide compared to the control which flowered late. There was no significant difference in yield traits among mutants and control, but the mutants and control of landraces could not survive the environment, therefore did not produce yield.

Table 2. Mean square interactive effect of genotype, concentration of sodium azide and growth stages on agronomic and yield characters of Bambara groundnut

\begin{tabular}{|c|c|c|c|c|}
\hline Source of variation & df & Number of days to flowering & Seed yield per plant $(\mathrm{g})$ & Seeds per pod \\
\hline Concentration & 4 & $34.51^{* * *}$ & $0.35^{\mathrm{ns}}$ & $0.01^{\mathrm{ns}}$ \\
\hline Genotype & 7 & $135.99^{* * *}$ & $1.63^{* * *}$ & $0.02^{* * *}$ \\
\hline Replicate & 2 & $2.29^{\text {ns }}$ & $8.32^{\mathrm{ns}}$ & $0.32 \mathrm{~ns}$ \\
\hline Week & 13 & $10809.03^{* * *}$ & $8.55^{* * *}$ & $0.33^{* * *}$ \\
\hline Genotype $\times$ concentration & 24 & $18.39^{* * *}$ & $0.43^{\mathrm{ns}}$ & $0.004^{\mathrm{ns}}$ \\
\hline Concentration $\times$ replicate & 8 & $18.12^{* * *}$ & $0.22^{\mathrm{ns}}$ & $0.01^{\mathrm{ns}}$ \\
\hline Concentration $\times$ week & 52 & $27.13^{* * *}$ & $0.33 \mathrm{~ns}$ & $0.01^{* * *}$ \\
\hline Genotype $\times$ replicate & 14 & $3.34^{\mathrm{ns}}$ & $1.73^{* * *}$ & $0.03^{* * *}$ \\
\hline Genotype $\times$ week & 91 & $113.7^{*}$ & $1.63^{* * *}$ & $0.03^{* * *}$ \\
\hline Week $\times$ replicate & 26 & $1.71^{\mathrm{ns}}$ & $5.50^{* *}$ & $0.21^{* * *}$ \\
\hline Genotype $\times$ concentration $\times$ replicate & 47 & $3.44^{\mathrm{ns}}$ & $0.46^{\mathrm{ns}}$ & $0.01^{\mathrm{ns}}$ \\
\hline Genotype $\times$ concentration $\times$ week & 309 & $14.20^{* * *}$ & $0.43^{\mathrm{ns}}$ & $0.004^{\mathrm{ns}}$ \\
\hline Concentration $\times$ week $\times$ replicate & 104 & $14.29^{* * *}$ & $0.21^{\mathrm{ns}}$ & $0.05^{\mathrm{ns}}$ \\
\hline Genotype $\times$ week $\times$ replicate & 182 & $2.48^{\mathrm{ns}}$ & $1.71^{* * *}$ & $0.03^{* * *}$ \\
\hline Error & 594 & 3.54 & 0.46 & 0.005 \\
\hline
\end{tabular}




\begin{tabular}{llllll}
\hline Source of variation & df & Number of days to flowering & Seed yield per plant $(\mathrm{g})$ & Seeds per pod & $\mathrm{P}$ \\
\hline Corrected total & 1477 & & & & \\
\hline
\end{tabular}

Note: ${ }^{*}=$ significant at $p<0.05,{ }^{* *}=$ highly significant at $p<0.01,{ }^{* * *}=$ highly significant at $p<0.001$

Table 3. Genotypic effect on germination and growth response of Bambara groundnut to sodium azide

\begin{tabular}{llllll}
\hline Genotype & Germination percentage (\%) & Plant length $(\mathrm{cm})$ & Leaf length $(\mathrm{cm})$ & Number of leaves & Number of branches \\
\hline TVSu 86 & $5.24^{\mathrm{a}}$ & $21.51^{\mathrm{d}}$ & $5.45^{\mathrm{d}}$ & $124.09^{\mathrm{a}}$ & $42.49^{\mathrm{a}}$ \\
TVSu 91 & $2.54^{\mathrm{bc}}$ & $12.80^{\mathrm{g}}$ & $2.98^{\mathrm{f}}$ & $57.74^{\mathrm{d}}$ & $19.63^{\mathrm{e}}$ \\
TVSu 186 & $4.77^{\mathrm{a}}$ & $26.21^{\mathrm{a}}$ & $7.11^{\mathrm{a}}$ & $117.78^{\mathrm{ab}}$ & $41.03^{\mathrm{ab}}$ \\
TVSu 235 & $4.92^{\mathrm{a}}$ & $25.08^{\mathrm{b}}$ & $6.07^{\mathrm{c}}$ & $118.28^{\mathrm{ab}}$ & $40.20^{\mathrm{ab}}$ \\
TVSu 242 & $2.38^{\mathrm{bc}}$ & $23.00^{\mathrm{c}}$ & $5.95^{\mathrm{c}}$ & $73.46^{\mathrm{c}}$ & $26.02^{\mathrm{c}}$ \\
TVSu 350 & $3.81^{\mathrm{ab}}$ & $25.53^{\mathrm{ab}}$ & $6.69^{\mathrm{b}}$ & $110.19^{\mathrm{b}}$ & $38.65^{\mathrm{b}}$ \\
Abia & $1.59^{\mathrm{c}}$ & $18.76^{\mathrm{f}}$ & $4.73^{\mathrm{e}}$ & $68.52^{\mathrm{c}}$ & $23.28^{\mathrm{d}}$ \\
Enugu & $4.76^{\mathrm{a}}$ & $20.00^{\mathrm{e}}$ & $4.92^{\mathrm{e}}$ & $43.64^{\mathrm{e}}$ & $15.05^{\mathrm{f}}$ \\
\hline
\end{tabular}

Means with different letter within a column differ significantly at $p<0.05$

Table 4. Effect of sodium azide concentrations on growth of Bambara groundnut

\begin{tabular}{llllll}
\hline Sodium azide concentrations $(\%)$ & Germination $(\%)$ & Plant length $(\mathrm{cm})$ & Leaf length $(\mathrm{cm})$ & Number of leaves & Number \\
\hline $0.00($ Control) & $4.37^{\mathrm{a}}$ & $25.10^{\mathrm{a}}$ & $6.33^{\mathrm{a}}$ & $108.46^{\mathrm{a}}$ & $37.51^{\mathrm{a}}$ \\
0.01 & $3.74^{\mathrm{ab}}$ & $22.41^{\mathrm{b}}$ & $5.84^{\mathrm{b}}$ & $93.12^{\mathrm{b}}$ & $32.77^{\mathrm{b}}$ \\
0.03 & $3.18^{\mathrm{b}}$ & $20.12^{\mathrm{c}}$ & $5.18^{\mathrm{c}}$ & $83.35^{\mathrm{c}}$ & $28.30^{\mathrm{c}}$ \\
0.05 & $3.86^{\mathrm{ab}}$ & $23.03^{\mathrm{b}}$ & $5.83^{\mathrm{b}}$ & $95.48^{\mathrm{b}}$ & $32.59^{\mathrm{b}}$ \\
0.07 & $2.98^{\mathrm{b}}$ & $18.15^{\mathrm{d}}$ & $4.58^{\mathrm{d}}$ & $93.38^{\mathrm{b}}$ & $32.36^{\mathrm{b}}$ \\
\hline
\end{tabular}

Means with different letter within a column differ significantly at $p<0.05$

Mutant seeds had significant increase in protein content. The concentration of $0.05 \%$ sodium azide had the highest protein content $(19.12 \%)$, while control had (18.5\%). The increase in protein content at higher concentration of sodium azide could be due to increase in auxin level. Similar results were observed in French bean by Mahamuneet al . (2017).

Table 5. Genotypic effects on protein contents, agronomic and yield of Bambara groundnut induced with sodium azide

\begin{tabular}{llllll}
\hline Genotype & Number of days to flowering & Seed yield per plant $(\mathrm{g})$ & Seeds per pod & Pod yield per plant $(\mathrm{g})$ & Number of \\
\hline TVSu 86 & $2.86^{\mathrm{cd}}$ & $0.17^{\mathrm{ab}}$ & $0.03^{\mathrm{a}}$ & $0.22^{\mathrm{abc}}$ & $0.32^{\mathrm{ab}}$ \\
TVSu 91 & $2.04^{\mathrm{e}}$ & $0.02^{\mathrm{b}}$ & $0.01^{\mathrm{bc}}$ & $0.03^{\mathrm{dc}}$ & $0.06^{\mathrm{bc}}$ \\
TVSu 186 & $3.44^{\mathrm{b}}$ & $0.05^{\mathrm{b}}$ & $0.02^{\mathrm{ab}}$ & $0.08^{\mathrm{bcd}}$ & $0.14^{\mathrm{bc}}$ \\
TVSu 235 & $2.49^{\mathrm{d}}$ & $0.25^{\mathrm{a}}$ & $0.03^{\mathrm{a}}$ & $0.37^{\mathrm{a}}$ & $0.50^{\mathrm{a}}$ \\
TVSu 242 & $3.59^{\mathrm{b}}$ & $0.02^{\mathrm{b}}$ & $0.01^{\mathrm{bc}}$ & $0.04^{\mathrm{dc}}$ & $0.08^{\mathrm{bc}}$ \\
TVSu 350 & $3.21^{\mathrm{bc}}$ & $0.14^{\mathrm{ab}}$ & $0.02^{\mathrm{ab}}$ & $0.25^{\mathrm{ab}}$ & $0.46^{\mathrm{a}}$ \\
Abia & $1.89^{\mathrm{e}}$ & $0.00^{\mathrm{b}}$ & $0.00^{\mathrm{c}}$ & $0.02^{\mathrm{dc}}$ & $0.00^{\mathrm{c}}$ \\
Enugu & $6.76^{\mathrm{a}}$ & $0.00^{\mathrm{b}}$ & $0.00^{\mathrm{c}}$ & $0.00^{\mathrm{d}}$ & $0.00^{\mathrm{c}}$ \\
\hline
\end{tabular}


Means with different letter within a column differ significantly at $p<0.05$

Table 6. Effect of sodium azide concentrations on protein contents, agronomic and yield characters of Bambara groundnut

\begin{tabular}{lllll}
\hline Sodium azide concentrations & Number of days to flowering & Seed yield per plant $(\mathrm{g})$ & Seeds per pod & Pod yield per plan \\
\hline $0.00 \%($ Control $)$ & $3.49^{\mathrm{a}}$ & $0.12^{\mathrm{a}}$ & $0.02^{\mathrm{a}}$ & $0.22^{\mathrm{a}}$ \\
$0.01 \%$ & $3.04^{\mathrm{b}}$ & $0.07^{\mathrm{a}}$ & $0.02^{\mathrm{a}}$ & $0.11^{\mathrm{a}}$ \\
$0.03 \%$ & $2.60^{\mathrm{c}}$ & $0.05^{\mathrm{a}}$ & $0.01^{\mathrm{a}}$ & $0.20^{\mathrm{a}}$ \\
$0.05 \%$ & $3.15^{\mathrm{b}}$ & $0.10^{\mathrm{a}}$ & $0.02^{\mathrm{a}}$ & $0.16^{\mathrm{a}}$ \\
$0.07 \%$ & $2.08^{\mathrm{d}}$ & $0.10^{\mathrm{a}}$ & $0.00^{\mathrm{a}}$ & $0.11^{\mathrm{a}}$ \\
\hline
\end{tabular}

Means with different letter within a column differ significantly at $p<0.05$

Table 7. Correlation coefficient of growth, agronomic and yield characters of Bambara groundnut induced with sodium azide.

\begin{tabular}{|c|c|c|c|c|c|c|c|c|c|c|c|c|}
\hline & G $\%$ & NB & $\mathrm{PH}$ & NL & LL & LW & $\mathrm{DF}$ & $\mathrm{PP}$ & NS & PY & SY & $\mathrm{S} / \mathrm{P}$ \\
\hline \multicolumn{13}{|l|}{ Germination \% } \\
\hline Number of branches & -0.24 & & & & & & & & & & & \\
\hline Plant height & 0.03 & $0.51^{*}$ & & & & & & & & & & \\
\hline Number of leaves & -0.23 & $0.98^{* *}$ & $0.50^{*}$ & & & & & & & & & \\
\hline Leaf length & 0.04 & 0.48 & $0.85^{* *}$ & 0.47 & & & & & & & & \\
\hline Leaf width & -0.05 & 0.45 & $0.74^{* *}$ & 0.45 & $0.71^{* *}$ & & & & & & & \\
\hline Days to flowering & -0.05 & 0.10 & 0.04 & 0.07 & 0.03 & 0.05 & & & & & & \\
\hline Pods per plant & -0.024 & 0.09 & 0.03 & 0.07 & 0.03 & 0.04 & 0.35 & & & & & \\
\hline Number of seeds & -0.02 & 0.09 & 0.03 & 0.07 & 0.03 & 0.04 & 0.35 & $0.99^{* *}$ & & & & \\
\hline Pod yield (g) & -0.02 & 0.08 & 0.02 & 0.07 & 0.03 & 0.04 & 0.33 & $0.96^{* *}$ & $0.96^{* *}$ & & & \\
\hline Seed yield (g) & -0.02 & 0.07 & 0.02 & 0.06 & 0.02 & 0.03 & 0.29 & $0.89^{* *}$ & $0.90^{* *}$ & $0.94^{* *}$ & & \\
\hline Seeds per pod & -0.03 & 0.08 & 0.03 & 0.06 & 0.03 & 0.05 & 0.38 & $0.85^{* *}$ & $0.85^{* *}$ & $0.86^{* *}$ & $0.84^{* *}$ & \\
\hline Genotype & -0.04 & -0.12 & 0.18 & -0.12 & 0.18 & -0.12 & 0.21 & 0.25 & 0.01 & -0.01 & -0.02 & -0.04 \\
\hline Concentration & -0.02 & -0.06 & -0.23 & -0.06 & -0.21 & -0.22 & 0.02 & -0.02 & -0.02 & -0.02 & -0.01 & -0.02 \\
\hline Week & -0.37 & $0.51^{*}$ & 0.11 & 0.49 & 0.05 & 0.16 & 0.40 & 0.18 & 0.18 & 0.17 & 0.16 & 0.20 \\
\hline Replicates & -0.00 & 0.01 & 0.04 & 0.02 & 0.01 & 0.01 & 0.00 & -0.12 & -0.12 & -0.12 & -0.11 & -0.14 \\
\hline
\end{tabular}

Note: ${ }^{*}=$ related (correlated) at $p<0.05,{ }^{* *}=$ strongly related at $p<0.01 . G \%=$ Germination percentage, $N B=$ Number of branches, $P H=$ Plant height, $N L=$ Number of leaves, $L L=$ Leaf length, $L W=$ Leaf width, $D F=$ Days to flowering, $P P=$ Pods per plant, $N S=$ Number of seeds, $P Y=$ Pod yield, $S Y=$ Seed yield, $S / P=$ Seeds per pod, $G=$ Genotype, $C=$ Concentration, $W=$ Week and $R=$ Replicate

The SDS-PAGE analysis revealed the presence of polypeptide bands in mutants which could be due to the damaging effect of the mutagen on different genes responsible for the polypeptide subunits. Similar occurrence had also been reported in Cowpea by Odeigah et al.(1998) and Mahamune et al . (2017).

The consumption of any legumes may result in flatulence and constipation which could be attributed to higher concentration of sodium azide in pesticide used in controlling pest damaging field crops (Ilbas et al., 2005).

\section{CONCLUSION}

The concentration of $0.07 \%$ Sodium azide mutagen reduced germination, growth and yield but increased the protein content of Bambara groundnut compared with control.TVSu-235 and TVSu-350 genotypes had 
higher tolerance and yield response to sodium azide concentrations therefore; they could be further utilised in crop improvement. The growth and yield traits should also be encouraged in mutagenic breeding of other leguminous crops.

\section{REFERENCES}

Asad, A., Koj, Y., Deka, U.Kr., \& Tomar , S.M.S.(2014) . Effect of Sodium Azide on seed

Germination and Related Agro-Metrical traits in $\mathrm{M}_{1}$ lentil (Lens culinaries

Medik.) Generation. World journal of Agricultural sciences, 10 (3): 95- 102.

DOI:10.5829/idosi.wjas.2014.10.3.8582

Aremu, M.O., Olaofe, O., \& Akintayo, E.T.(2006 ). Chemical composition and

physiochemical characteristics of two varieties of Bambara groundnut (Vigna

subterrenea ) flours. Journal of Applied Sciences , 6(9):1900-1903.

Agbolade, J.O., Olawuyi, O.J., Bello, O.B., Oluseye, O.D., \& Komolafe, R.J.S. (2016).

Genetic diversity and correlated response to selection of grain and associated characters in maize (Zea mays (L.). Journal of Basic and Applied research international Science, 13(1): 56-61. Retrieved from

http://www.ikprress.org/index.php/JOBARI/article/view/3410

AOAC. Official Method of Analysis. 15th ed. (1996). Association of Official Analytical

Chemist, Washington DC - Science and Education Publishing. Pp. 550-575.

Arulbalachandran, D., Mullainathan, L., \& Velu, S. (2009). Screening of mutants in black gram (Vigna mungo (L.) Hepper) with effect of DES and $\mathrm{COH}$ in $\mathrm{M}_{2}$ generation. Journal of Phytology, 1(4): 213-218.

Asgharipour, M.R., \& Rafiei, M. (2011). Effect of Salinity on Germination and Seedling

Growth of Lentils. Australian Journal of Basic Applied Science, 5(11):2002-2004.

Bamshaiye, O. M., Adegbol, J.A., \& Bamishaiye, E.I. (2011). Bambara groundnut: An Under- utilized nut in Africa. Adv. In Agri. Biotechnology, 1: 60-72.

Cokkizgin, A. \& Cokkizgin, H. (2010). Effect of Lead $\left(\mathrm{PbCl}_{2}\right)$ stress on germination of Lentil (Lens culinaries Medic.) Lines. African Journal of Biotechnology , 9(50):8608-8612.

Descriptors for Bambara groundnut (Vigna subterranea (L).Verdc). (2020). IPGRI, BAMNET, publication

Ghafoor, A., Ahmad, Z., Qureshi, A. S., \& Bashir, M. (2002). Genetic relationship in Vigna mungo (L.) Hepper and V. radiate (L.) R.Wilczek based on morphological traits and SDS-PAGE. Euphytica,123: 367378.

Ilbas, A.I., Eroglu, Y., \& Eroglu, H. E. (2005). Effects of the application of different concentrations of $\mathrm{NaN}_{3}$ for different times on the morphological and cytogenetic characteristics of barley (Hordeum vulgare L.) seedlings. Journal of Integrative Plant Biology , 47: 1101-1106. doi: 10.1111/j.1744-7909.2005.00137.x.

Iqbal, S. M., Ghafoor, A., \& Ayub, N. (2005). Relationship between SDS-PAGE markers and Ascochyta blight in chickpea. Pakistan Journal of Botany , 37(1):87-96 
Mahamune, S. E, Sangle, S. M and Kothekar, V.S. 2017. Protein profile studies in French bean mutants. Bioscience Discovery 8(3): 328-334.

Mennella, G., Onofaro, S.V., Tonini, A. \& Magnifico, V. (1999). Seed storage protein characterization of Solanum specied and of cultivars and androgenic lines of $S$. melongena L., by SDS-PAGE and AE-hpic. Seed Sci. Technol. 27:23-35.

Mensah, J. K., \& Obadoni, B. (2007). Effects of Sodium azide on yield parameters of groundnut (Arachis hypogaea L.). Afr. J. Biotechnol , 6(6): $668-671$.

Mostafa, G. G. (2011). Effect of sodium azide on the growth and variability induction in (Helianthus annuus L.). Intl. J. Plant Breed. Genet, 5(1): 76-85. doi: 10.3923/ijpbg.2011.76.85.

Olawuyi, O. J., Bello, O.B., Ntube, C.V., \& Akanmu, A.O. (2015). Progress from selection of some maize cultivars' response to drought in the derived savanna of Nigeria, Agrivita. J. Agric.Sc, 37(1): 8-17. doi: 10.17503/Agrivita-2015-37-1-p008-017.

Olawuyi, O. J., Bello, O. B \& Abioye, A. O. (2016). Mutagenic effects of Ultraviolet Radiation on growth and agronomic characters in maize cultivars. Mol. Plant Breeding, 7(1): 1-10.

Olawuyi, O. J., \& Okoli, S.O. (2017). Genetic variability on tolerance of maize (Zea mays L.) genotypes induced with sodium azide mutagen. Molecular Plant Breeding, 8(3): 27-37.

Odeigah, P.G.C. \& Osanyinpeju, A.O. (1998). Induced mutation in cowpea(Vigna unguiculata). J. Genet Breeding, 51: 126-132.

Weber, K. \& M. Osborn, 1975. Proteins and Sodium dodecyl sulphate. Molecular Weight determination on polyacrylamide Gels and related procedures: In The Proteins (Neurath H. and R.L. Hill, (Eds.) $3^{\text {rd }}$ Edn, Academic Press, New York, 1:179-223.

Wongpiyasatid, A., Chotechuen, S., Hormchan, P., Ngampongsai, S., \& Promcham, W. (2000). Induced mutations in Mungbean breeding: Regional yield trial of mungbean mutant lines. Kasetsart J. Nat. Sci. Res., 34: 443-449.

Table 1. Mean square interaction of genotype, concentrations of Sodium azide and growth stage on germination and growth characters of Bambara groundnut

\begin{tabular}{llllll}
\hline Source of variation & Df & Germination percentage & Plant height & Leaf length & Number of leaves \\
\hline Concentration & 4 & $9.69^{\mathrm{ns}}$ & $2121.02^{* * *}$ & $134.37^{* * *}$ & $42476.98^{* * *}$ \\
Genotype & 7 & $385.48^{* * *}$ & $3321.67^{* * *}$ & $274.36^{* * *}$ & $143861.73^{* * *}$ \\
Replicate & 2 & $2.32^{\mathrm{ns}}$ & $60.89^{\mathrm{ns}}$ & $11.92^{\mathrm{ns}}$ & $5453.61^{\mathrm{ns}}$ \\
Week & 13 & $17146.10^{* * *}$ & $34.80^{* * *}$ & $2.07^{\mathrm{ns}}$ & $91419.258^{* * *}$ \\
Genotype $\times$ concentration & 24 & $87.58^{\mathrm{ns}}$ & $892.50^{* * *}$ & $52.29^{* * *}$ & $26745.69^{* * *}$ \\
Concentration $\times$ replicate & 8 & $74.82^{\mathrm{ns}}$ & $120.31^{* * *}$ & $5.18^{* * *}$ & $25610.86^{* * *}$ \\
Concentration $\times$ week & 52 & $99.59^{* * *}$ & $30.14^{* * *}$ & $2.18^{* * *}$ & $1828.05^{* * *}$ \\
Genotype $\times$ replicate & 14 & $73.01^{\mathrm{ns}}$ & $66.85^{* * *}$ & $3.29^{* * *}$ & $3478.95^{* * *}$ \\
Genotype $\times$ week & 91 & $385.60^{* * *}$ & $47.53^{* * *}$ & $3.61^{* * *}$ & $3786.25^{* * *}$ \\
Week $\times$ replicate & 26 & $10.52^{\mathrm{ns}}$ & $11.59^{\mathrm{ns}}$ & $0.69^{\mathrm{ns}}$ & $539.00^{\mathrm{ns}}$ \\
Genotype $\times$ concentration $\times$ replicate & 47 & $45.91^{\mathrm{ns}}$ & $117.29^{* * *}$ & $10.33^{* * *}$ & $13250.09^{* * *}$ \\
Genotype $\times$ concentration $\times$ week & 312 & $87.58^{* * *}$ & $32.89^{* * *}$ & $3.05^{* * *}$ & $2166.30^{* *}$ \\
Concentration $\times$ week $\times$ replicate & 104 & $74.82^{* * *}$ & $15.29^{\mathrm{ns}}$ & $1.52^{*}$ & $1629.64^{* * *}$ \\
Genotype $\times$ week $\times$ replicate & 182 & $73.01^{* * *}$ & $18.88^{*}$ & $1.39^{\mathrm{ns}}$ & $1094.67^{\mathrm{ns}}$ \\
Error & 611 & 611 & 562 & 562 & 560 \\
Corrected total & 1497 & 1497 & 1446 & 1446 & 1444 \\
\hline
\end{tabular}


Note: ${ }^{*}=$ significant at $p<0.05,{ }^{* *}=$ highly significant at $p<0.01,{ }^{* * *}=$ highly significant at $p<0.001$

Table 2. Mean square interactive effect of genotype, concentration of sodium azide and growth stages on agronomic and yield characters of Bambara groundnut

\begin{tabular}{llllll}
\hline Source of variation & df & Number of days to flowering & Seed yield per plant $(\mathrm{g})$ & Seeds per pod & $\mathrm{P}$ \\
\hline Concentration & 4 & $34.51^{* * *}$ & $0.35^{\mathrm{ns}}$ & $0.01^{\mathrm{ns}}$ & $1.63^{* * *}$ \\
Genotype & 7 & $135.99^{* * *}$ & $8.32^{\mathrm{ns}}$ & $0.02^{* * *}$ & $3.32^{\mathrm{ns}}$ \\
Replicate & 2 & $2.29^{\mathrm{ns}}$ & $8.55^{* * *}$ & $0.33^{* * *}$ & 1 \\
Week & 13 & $10809.03^{* * *}$ & $0.43^{\mathrm{ns}}$ & $0.004^{\mathrm{ns}}$ & 0 \\
Genotype $\times$ concentration & 24 & $18.39^{* * *}$ & $0.22^{\mathrm{ns}}$ & $0.01^{\mathrm{ns}}$ & $0.33 \mathrm{~ns}$ \\
Concentration $\times$ replicate & 8 & $18.12^{* * *}$ & $1.73^{* * *}$ & $0.01^{* * *}$ & 1 \\
Concentration $\times$ week & 52 & $27.13^{* * *}$ & $1.63^{* * *}$ & $0.03^{* * *}$ & 3 \\
Genotype $\times$ replicate & 14 & $3.34^{\mathrm{ns}}$ & $5.50^{* *}$ & $0.03^{* * *}$ & 3 \\
Genotype $\times$ week & 91 & $113.7^{*}$ & $0.46^{\mathrm{ns}}$ & $0.21^{* * *}$ & 1 \\
Week $\times$ replicate & 26 & $1.71^{\mathrm{ns}}$ & $0.43^{\mathrm{ns}}$ & $0.01^{\mathrm{ns}}$ & $0.004^{\mathrm{ns}}$ \\
Genotype $\times$ concentration $\times$ replicate & 47 & $3.44^{\mathrm{ns}}$ & $0.21^{\mathrm{ns}}$ & $0.05^{\mathrm{ns}}$ \\
Genotype $\times$ concentration $\times$ week & 309 & $14.20^{* * *}$ & $1.71^{* * *}$ & $0.03^{* * *}$ & 0 \\
Concentration $\times$ week $\times$ replicate & 104 & $14.29^{* * *}$ & & 0.005 & 0 \\
Genotype $\times$ week $\times$ replicate & 182 & $2.48^{\mathrm{ns}}$ & & \\
Error & 594 & 3.54 & & & \\
Corrected total & 1477 & & &
\end{tabular}

Note: ${ }^{*}=$ significant at $p<0.05,{ }^{* *}=$ highly significant at $p<0.01,{ }^{* * *}=$ highly significant at $p<0.001$

Table 3. Genotypic effect on germination and growth response of Bambara groundnut to sodium azide

\begin{tabular}{llllll}
\hline Genotype & Germination percentage $(\%)$ & Plant length $(\mathrm{cm})$ & Leaf length $(\mathrm{cm})$ & Number of leaves & Number of branches \\
\hline TVSu 86 & $5.24^{\mathrm{a}}$ & $21.51^{\mathrm{d}}$ & $5.45^{\mathrm{d}}$ & $124.09^{\mathrm{a}}$ & $42.49^{\mathrm{a}}$ \\
TVSu 91 & $2.54^{\mathrm{bc}}$ & $12.80^{\mathrm{g}}$ & $2.98^{\mathrm{f}}$ & $57.74^{\mathrm{d}}$ & $19.63^{\mathrm{e}}$ \\
TVSu 186 & $4.77^{\mathrm{a}}$ & $26.21^{\mathrm{a}}$ & $7.11^{\mathrm{a}}$ & $117.78^{\mathrm{ab}}$ & $41.03^{\mathrm{ab}}$ \\
TVSu 235 & $4.92^{\mathrm{a}}$ & $25.08^{\mathrm{b}}$ & $6.07^{\mathrm{c}}$ & $118.28^{\mathrm{ab}}$ & $40.20^{\mathrm{ab}}$ \\
TVSu 242 & $2.38^{\mathrm{bc}}$ & $23.00^{\mathrm{c}}$ & $5.95^{\mathrm{c}}$ & $73.46^{\mathrm{c}}$ & $26.02^{\mathrm{c}}$ \\
TVSu 350 & $3.81^{\mathrm{ab}}$ & $25.53^{\mathrm{ab}}$ & $6.69^{\mathrm{b}}$ & $110.19^{\mathrm{b}}$ & $38.65^{\mathrm{b}}$ \\
Abia & $1.59^{\mathrm{c}}$ & $18.76^{\mathrm{f}}$ & $4.73^{\mathrm{e}}$ & $68.52^{\mathrm{c}}$ & $23.28^{\mathrm{d}}$ \\
Enugu & $4.76^{\mathrm{a}}$ & $20.00^{\mathrm{e}}$ & $4.92^{\mathrm{e}}$ & $43.64^{\mathrm{e}}$ & $15.05^{\mathrm{f}}$ \\
\hline
\end{tabular}

Means with different letter within a column differ significantly at $p<0.05$

Table 4. Effect of sodium azide concentrations on growth of Bambara groundnut

\begin{tabular}{llllll}
\hline Sodium azide concentrations $(\%)$ & Germination $(\%)$ & Plant length $(\mathrm{cm})$ & Leaf length $(\mathrm{cm})$ & Number of leaves & Number \\
\hline $0.00($ Control $)$ & $4.37^{\mathrm{a}}$ & $25.10^{\mathrm{a}}$ & $6.33^{\mathrm{a}}$ & $108.46^{\mathrm{a}}$ & $37.51^{\mathrm{a}}$ \\
0.01 & $3.74^{\mathrm{ab}}$ & $22.41^{\mathrm{b}}$ & $5.84^{\mathrm{b}}$ & $93.12^{\mathrm{b}}$ & $32.77^{\mathrm{b}}$ \\
0.03 & $3.18^{\mathrm{b}}$ & $20.12^{\mathrm{c}}$ & $5.18^{\mathrm{c}}$ & $83.35^{\mathrm{c}}$ & $28.30^{\mathrm{c}}$ \\
0.05 & $3.86^{\mathrm{ab}}$ & $23.03^{\mathrm{b}}$ & $5.83^{\mathrm{b}}$ & $95.48^{\mathrm{b}}$ & $32.59^{\mathrm{b}}$ \\
0.07 & $2.98^{\mathrm{b}}$ & $18.15^{\mathrm{d}}$ & $4.58^{\mathrm{d}}$ & $93.38^{\mathrm{b}}$ & $32.36^{\mathrm{b}}$ \\
\hline
\end{tabular}


Means with different letter within a column differ significantly at $p<0.05$

Table 5. Genotypic effects on protein contents, agronomic and yield of Bambara groundnut induced with sodium azide

\begin{tabular}{llllll}
\hline Genotype & Number of days to flowering & Seed yield per plant $(\mathrm{g})$ & Seeds per pod & Pod yield per plant $(\mathrm{g})$ & Number of \\
\hline TVSu 86 & $2.86^{\mathrm{cd}}$ & $0.17^{\mathrm{ab}}$ & $0.03^{\mathrm{a}}$ & $0.22^{\mathrm{abc}}$ & $0.32^{\mathrm{ab}}$ \\
TVSu 91 & $2.04^{\mathrm{e}}$ & $0.02^{\mathrm{b}}$ & $0.01^{\mathrm{bc}}$ & $0.03^{\mathrm{dc}}$ & $0.06^{\mathrm{bc}}$ \\
TVSu 186 & $3.44^{\mathrm{b}}$ & $0.05^{\mathrm{b}}$ & $0.02^{\mathrm{ab}}$ & $0.08^{\mathrm{bcd}}$ & $0.14^{\mathrm{bc}}$ \\
TVSu 235 & $2.49^{\mathrm{d}}$ & $0.25^{\mathrm{a}}$ & $0.03^{\mathrm{a}}$ & $0.37^{\mathrm{a}}$ & $0.50^{\mathrm{a}}$ \\
TVSu 242 & $3.59^{\mathrm{b}}$ & $0.02^{\mathrm{b}}$ & $0.01^{\mathrm{bc}}$ & $0.04^{\mathrm{dc}}$ & $0.08^{\mathrm{bc}}$ \\
TVSu 350 & $3.21^{\mathrm{bc}}$ & $0.14^{\mathrm{ab}}$ & $0.02^{\mathrm{ab}}$ & $0.25^{\mathrm{ab}}$ & $0.46^{\mathrm{a}}$ \\
Abia & $1.89^{\mathrm{e}}$ & $0.00^{\mathrm{b}}$ & $0.00^{\mathrm{c}}$ & $0.02^{\mathrm{dc}}$ & $0.00^{\mathrm{c}}$ \\
Enugu & $6.76^{\mathrm{a}}$ & $0.00^{\mathrm{b}}$ & $0.00^{\mathrm{c}}$ & $0.00^{\mathrm{d}}$ & $0.00^{\mathrm{c}}$ \\
\hline
\end{tabular}

Means with different letter within a column differ significantly at $p<0.05$

Table 6. Effect of sodium azide concentrations on protein contents, agronomic and yield characters of Bambara groundnut

\begin{tabular}{lllll}
\hline Sodium azide concentrations & Number of days to flowering & Seed yield per plant $(\mathrm{g})$ & Seeds per pod & Pod yield per plan \\
\hline $0.00 \%($ Control $)$ & $3.49^{\mathrm{a}}$ & $0.12^{\mathrm{a}}$ & $0.02^{\mathrm{a}}$ & $0.22^{\mathrm{a}}$ \\
$0.01 \%$ & $3.04^{\mathrm{b}}$ & $0.07^{\mathrm{a}}$ & $0.02^{\mathrm{a}}$ & $0.11^{\mathrm{a}}$ \\
$0.03 \%$ & $2.60^{\mathrm{c}}$ & $0.05^{\mathrm{a}}$ & $0.01^{\mathrm{a}}$ & $0.20^{\mathrm{a}}$ \\
$0.05 \%$ & $3.15^{\mathrm{b}}$ & $0.10^{\mathrm{a}}$ & $0.02^{\mathrm{a}}$ & $0.16^{\mathrm{a}}$ \\
$0.07 \%$ & $2.08^{\mathrm{d}}$ & $0.10^{\mathrm{a}}$ & $0.00^{\mathrm{a}}$ & $0.11^{\mathrm{a}}$ \\
\hline
\end{tabular}

Means with different letter within a column differ significantly at $p<0.05$

Table 7. Correlation coefficient of growth, agronomic and yield characters of Bambara groundnut induced with sodium azide.

\begin{tabular}{|c|c|c|c|c|c|c|c|c|c|c|c|c|}
\hline & G \% & NB & $\mathrm{PH}$ & NL & LL & LW & $\mathrm{DF}$ & $\mathrm{PP}$ & NS & PY & SY & $\mathrm{S} / \mathrm{P}$ \\
\hline \multicolumn{13}{|l|}{ Germination \% } \\
\hline Number of branches & -0.24 & & & & & & & & & & & \\
\hline Plant height & 0.03 & $0.51^{*}$ & & & & & & & & & & \\
\hline Number of leaves & -0.23 & $0.98^{* *}$ & $0.50^{*}$ & & & & & & & & & \\
\hline Leaf length & 0.04 & 0.48 & $0.85^{* *}$ & 0.47 & & & & & & & & \\
\hline Leaf width & -0.05 & 0.45 & $0.74^{* *}$ & 0.45 & $0.71^{* *}$ & & & & & & & \\
\hline Days to flowering & -0.05 & 0.10 & 0.04 & 0.07 & 0.03 & 0.05 & & & & & & \\
\hline Pods per plant & -0.024 & 0.09 & 0.03 & 0.07 & 0.03 & 0.04 & 0.35 & & & & & \\
\hline Number of seeds & -0.02 & 0.09 & 0.03 & 0.07 & 0.03 & 0.04 & 0.35 & $0.99^{* *}$ & & & & \\
\hline Pod yield (g) & -0.02 & 0.08 & 0.02 & 0.07 & 0.03 & 0.04 & 0.33 & $0.96^{* *}$ & $0.96^{* *}$ & & & \\
\hline Seed yield (g) & -0.02 & 0.07 & 0.02 & 0.06 & 0.02 & 0.03 & 0.29 & $0.89^{* *}$ & $0.90^{* *}$ & $0.94^{* *}$ & & \\
\hline Seeds per pod & -0.03 & 0.08 & 0.03 & 0.06 & 0.03 & 0.05 & 0.38 & $0.85^{* *}$ & $0.85^{* *}$ & $0.86^{* *}$ & $0.84^{* *}$ & \\
\hline Genotype & -0.04 & -0.12 & 0.18 & -0.12 & 0.18 & -0.12 & 0.21 & 0.25 & 0.01 & -0.01 & -0.02 & -0.04 \\
\hline Concentration & -0.02 & -0.06 & -0.23 & -0.06 & -0.21 & -0.22 & 0.02 & -0.02 & -0.02 & -0.02 & -0.01 & -0.02 \\
\hline Week & -0.37 & $0.51^{*}$ & 0.11 & 0.49 & 0.05 & 0.16 & 0.40 & 0.18 & 0.18 & 0.17 & 0.16 & 0.20 \\
\hline Replicates & -0.00 & 0.01 & 0.04 & 0.02 & 0.01 & 0.01 & 0.00 & -0.12 & -0.12 & -0.12 & -0.11 & -0.14 \\
\hline
\end{tabular}


Note: ${ }^{*}=$ related (correlated) at $p<0.05,{ }^{*}=$ strongly related at $p<0.01 . G \%=$ Germination percentage, $N B=$ Number of branches, $P H=$ Plant height,$N L=$ Number of leaves, $L L=$ Leaf length, $L W=$ Leaf width, $D F=$ Days to flowering, $P P=$ Pods per plant, $N S=$ Number of seeds, $P Y=$ Pod yield, $S Y=$ Seed yield, $S / P=$ Seeds per pod, $G=$ Genotype, $C=$ Concentration, $W=$ Week and $R=$ Replicate

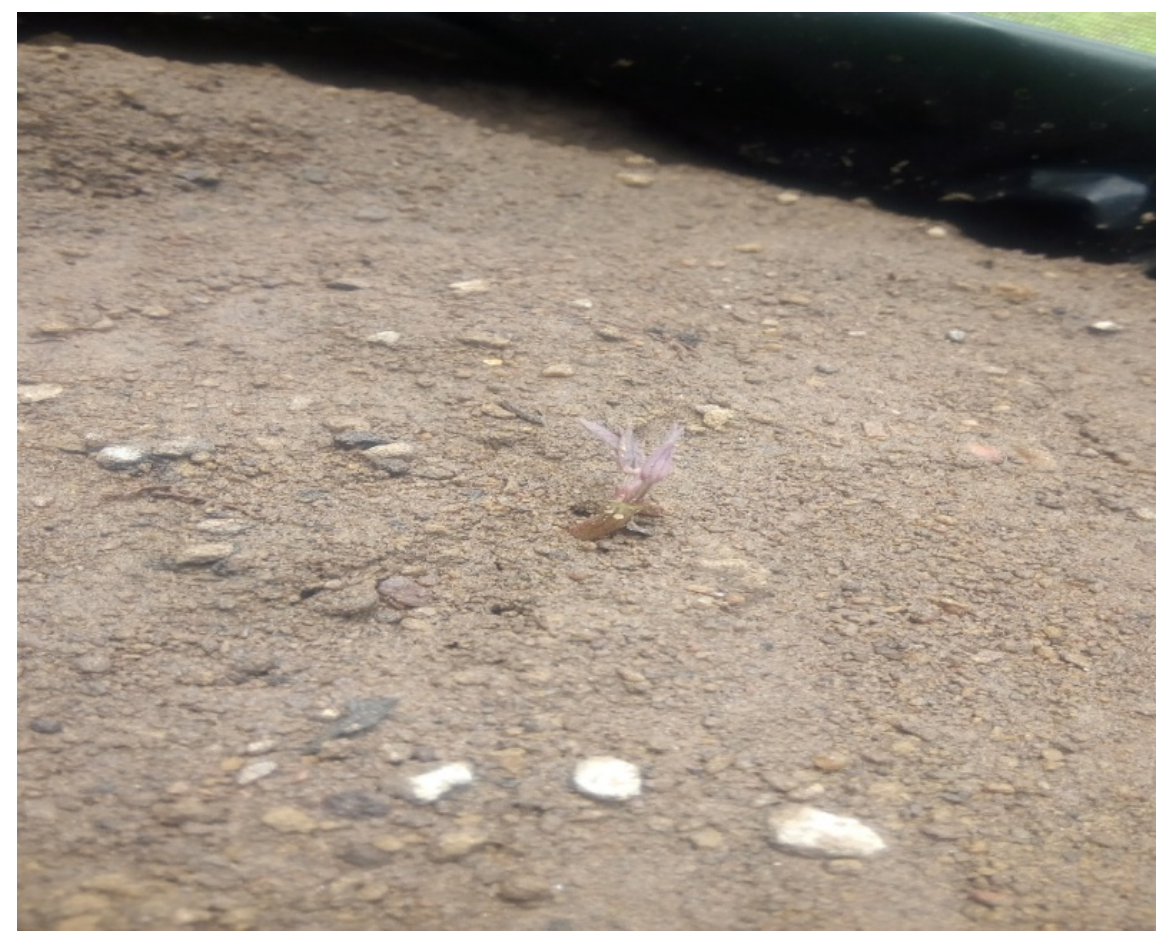




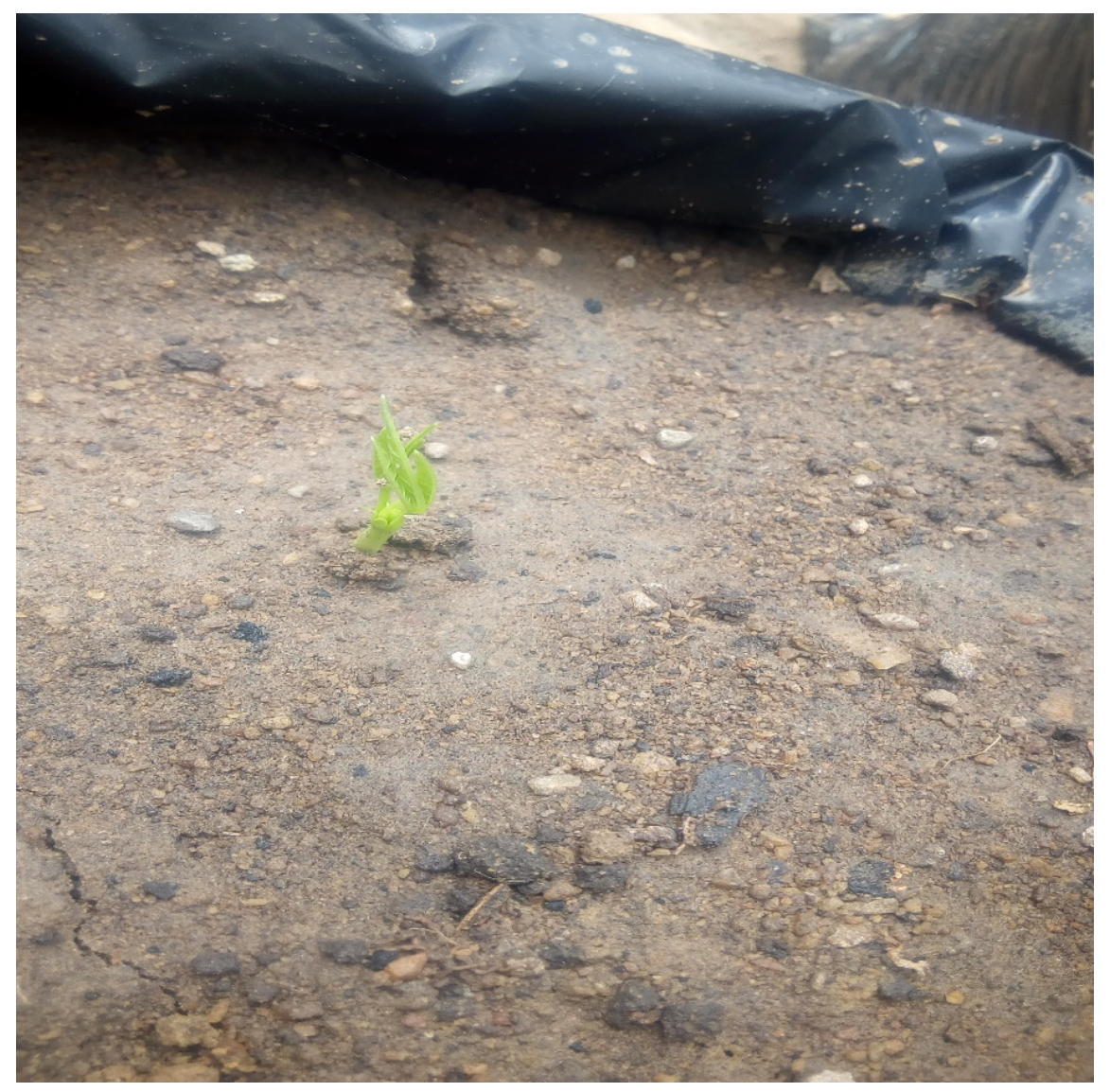

Plate 1a. TVSu-186 (0.07\%) induced with Plate 1b . TVSu-186 (0.00\%) control Sodium azide showing purple seedling showing normal colour 


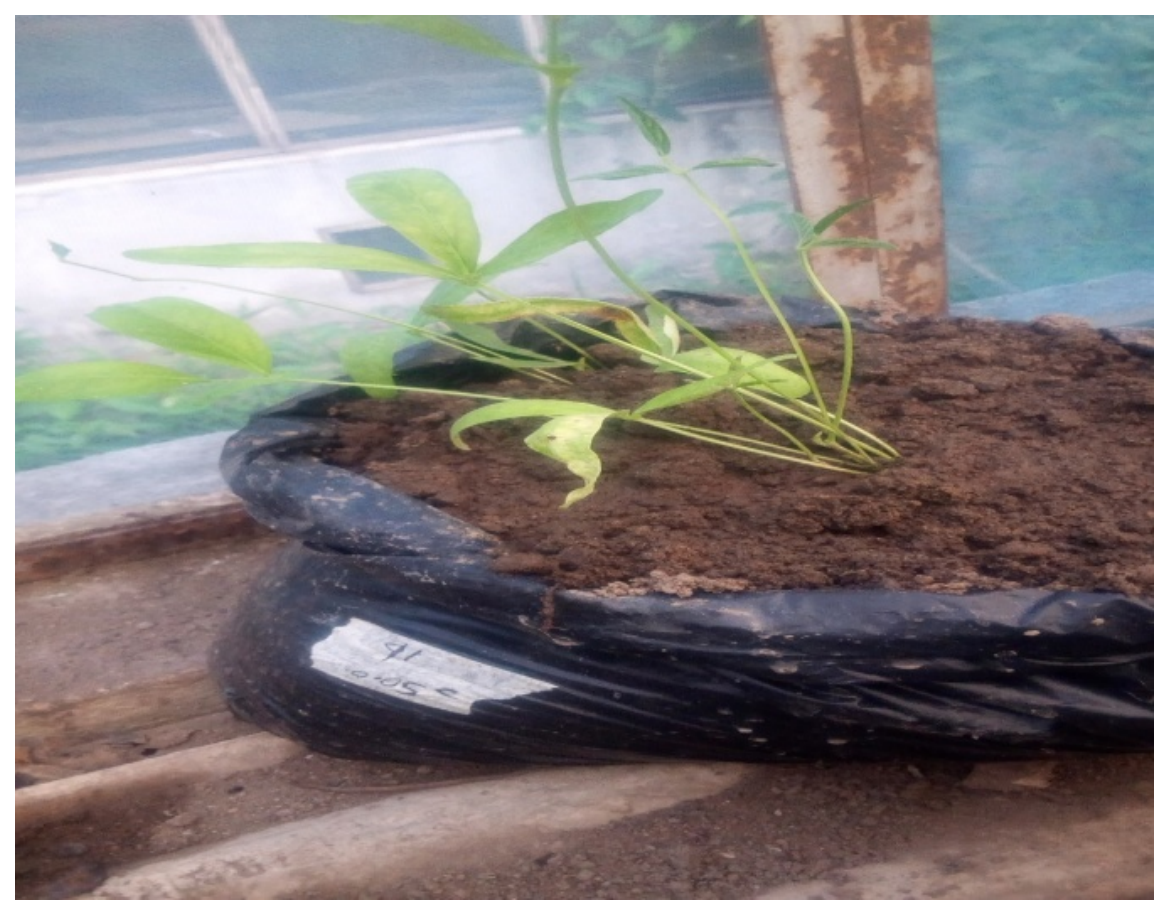




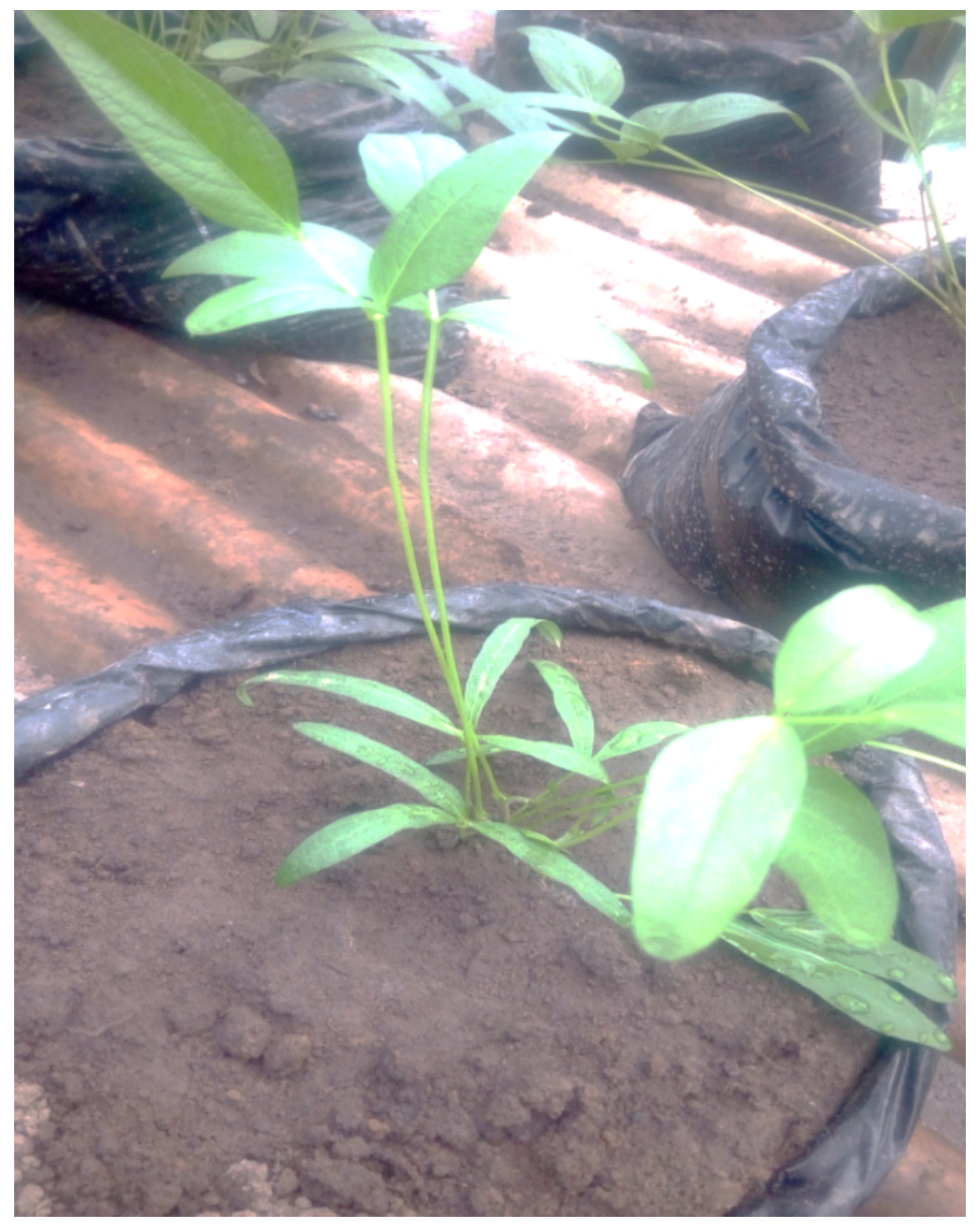

Plate 2a. Abia landraces induced with Plate 2b .TVSu-91 induced with $0.05 \%$ $0.05 \%$ Sodium azide showed creeping Sodium azide had two different leaves shape stem and yellow colour. in a single plant stand. 


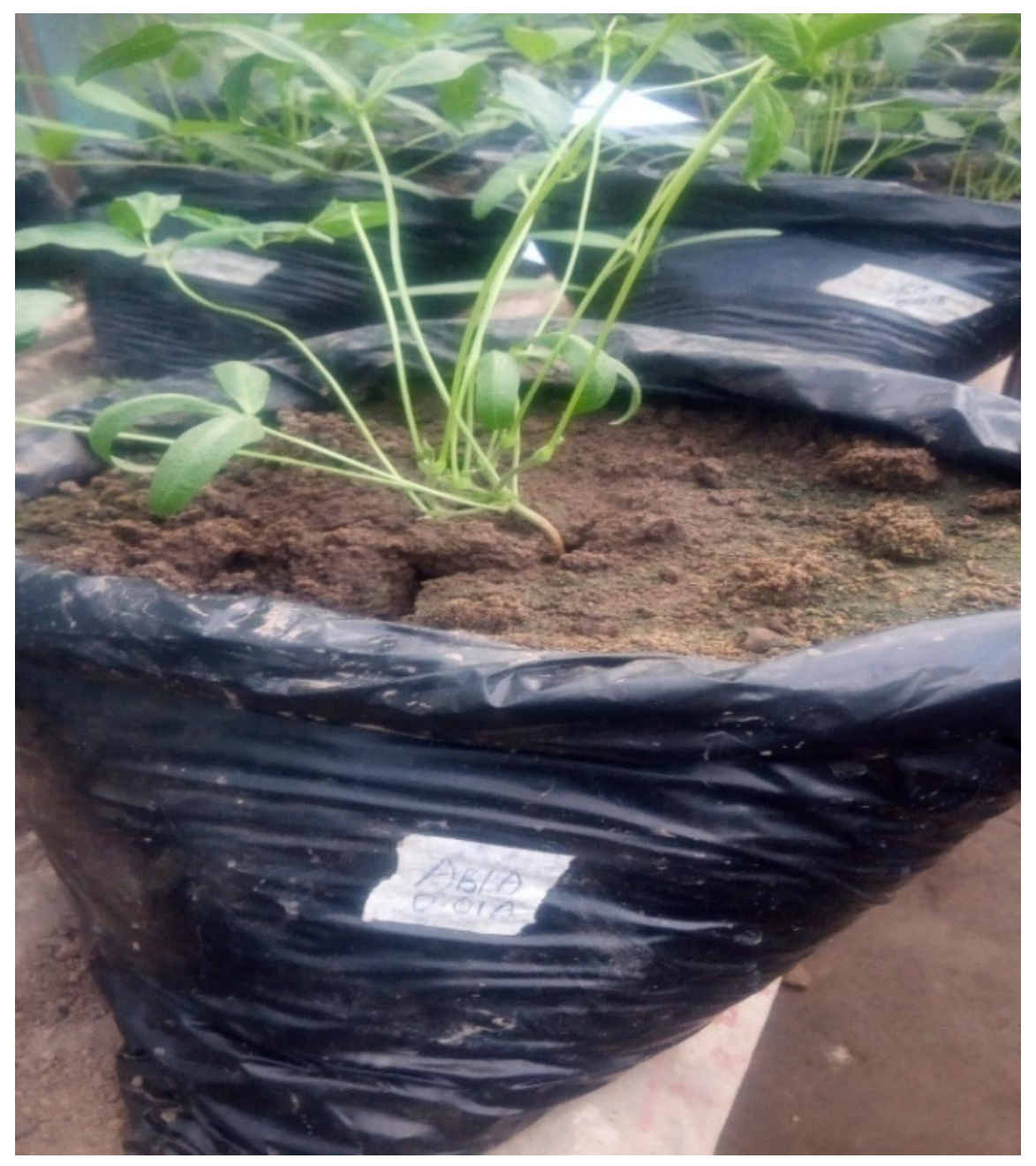




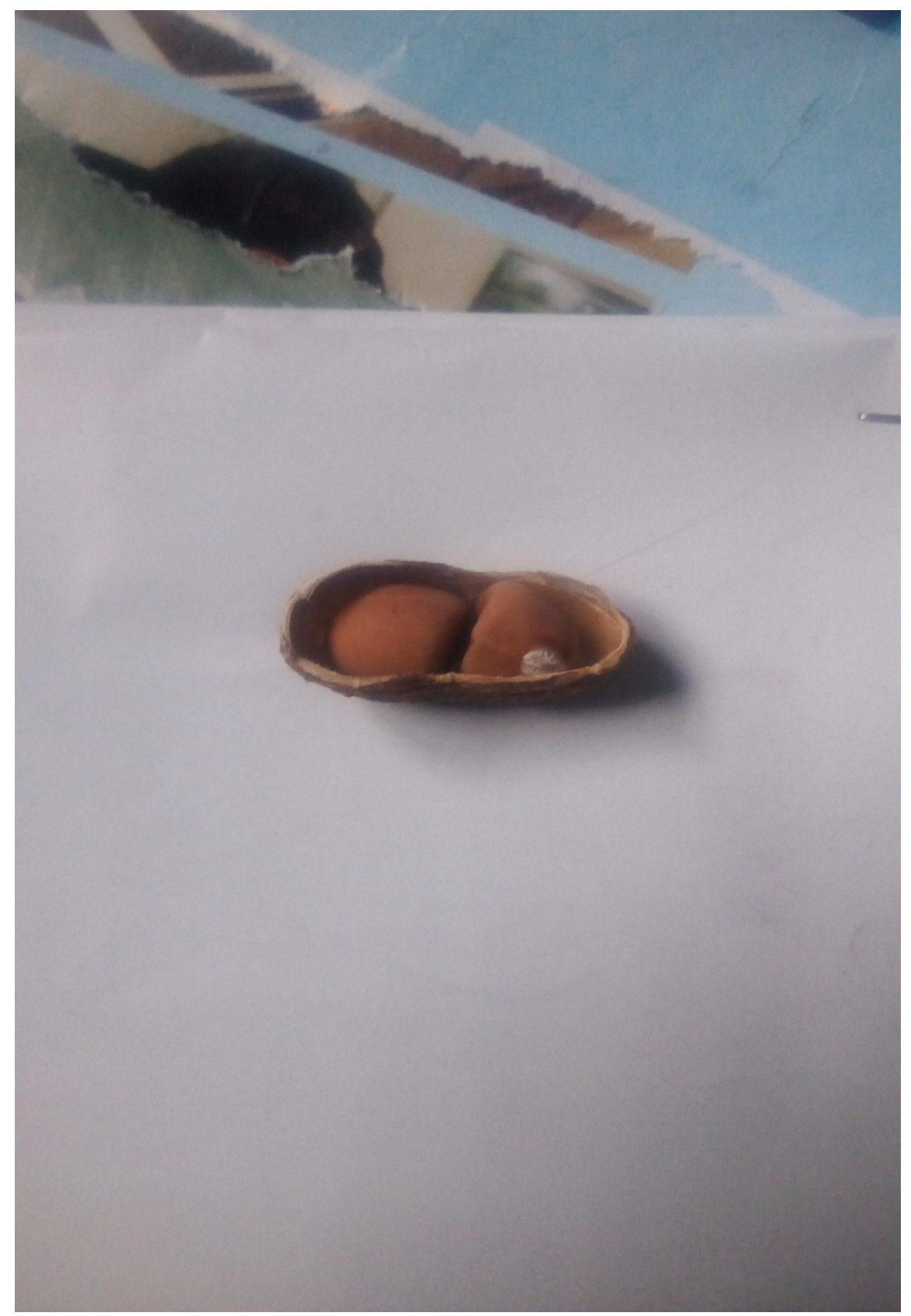

Plate 3. Abia landrace induced with $0.01 \%$ Plate 4.TVSu-86 (0.07\%) produced mutants Sodium azide showed crinkled (rough and with two seeds per pod which was not twisting) leaves. observed in other concentrations. 


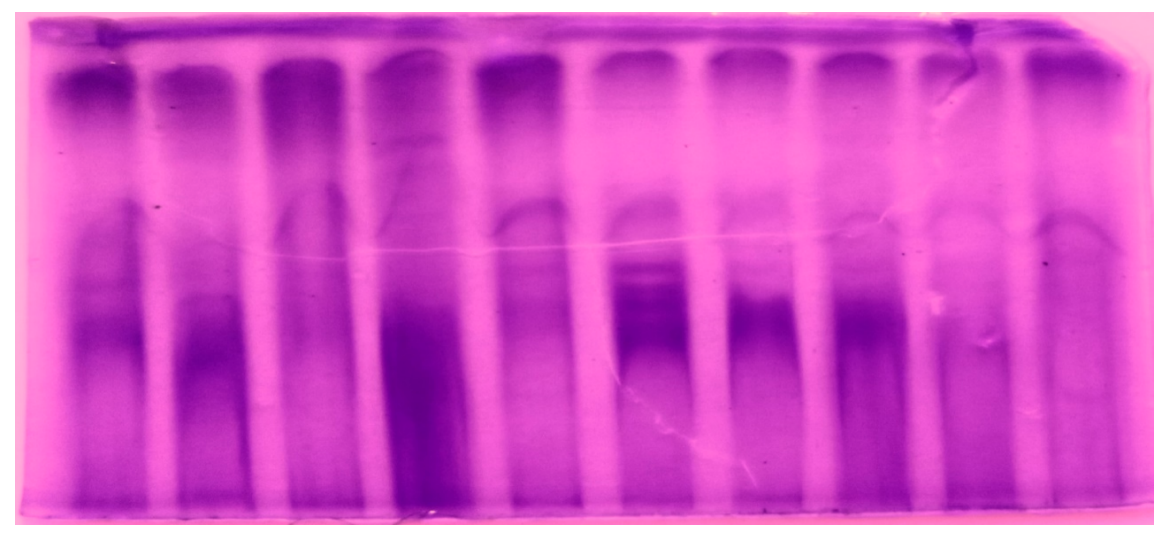

Plate 5 . SDS-polyacrylamide gel electrophoregram of seed protein profile.

\section{Hosted file}

ArtiCle Figures.pdf available at https://authorea.com/users/361769/articles/483110-studieson-bambara-groundnut-vigna-subterranea-l-verdc-induced-with-sodium-azide-using-sodiumdodecyl-sulphate-polyacrlyamide-gel-electrophoresis-sds-page 